\title{
Molecular Mechanisms of Mismatch Repair Genes in Cancer - A Brief Review
}

\author{
Shilpa $\mathrm{V}^{* 1}$ and Lakshmi $\mathrm{K}^{1,2}$ \\ ${ }^{1}$ Department of Biochemistry, Kidwai Memorial Institute of Oncology, Bangalore \\ ${ }^{2}$ Department of Biochemistry, Sri Shankara Cancer Hospital \& Research Centre, Bangalore
}

*Corresponding author: Shilpa V, Department of Biochemistry, Kidwai Memorial Institute of Oncology, Dr. M. H. Marigowda Road, Bangalore-560029, India, Fax: 080-26560723, Tel: 080-26094072, E-mail: shilpachadaga@gmail.com

Citation: Shilpa V, Lakshmi K (2014) Molecular Mechanisms of Mismatch Repair Genes in Cancer - A Brief Review. J Proteomics Genomics 1(1): 101

Received Date: April 24, 2014 Accepted Date: July 01, 2014 Published Date: July 02, 2014

\begin{abstract}
The DNA mismatch repair (MMR) system is necessary for the maintenance of genomic stability. The MMR system promotes genomic fidelity by repairing base-base mismatches, insertion-deletion loops (IDLs) and heterologies generated during DNA replication and recombination. Failure to accomplish these functions may lead to cancer and are associated with tumor prone phenotypes. MMR proteins coordinate a complex network of physical and functional interactions and are also involved in activation of cell-cycle check point and induction of apoptosis during DNA damage. They also play a role in cell death by alkylating agents and other chemotherapeutic drugs. Inactivation of MMR genes by mutations or epigenetic silencing in hereditary and sporadic cancers is associated with mutator phenotype and inhibition of apoptosis. A deeper understanding of the molecular mechanism and functional interactions of MMR proteins will lead to the development of more effective cancer prevention and treatment strategies.

Keywords: DNA damage; DNA repair; Epigenetic regulation; Genome instability; MMR; MSI

Abbreviations: AML: Acute myeloid leukemia; BER: Base excision repairtal cance; BLM: Bloom syndrome helicase; BASC: BRCA1 associated genome surveillance complex; CRC: Colorectal cancer; DA: Damage avoidance; DDB: Double stranded break; EXO: Exonuclease; HNPCC: Hereditary nonpolyposis colon cancer; IDL: Insertion-deletion loop; MBD: 5methyl cytosine binding domain; MGMT: Methyl guanosine methyl transferase; MSI: Microsatellite instability; MSI- H: Microsatellite instability high; MSI-L: Microsatellite instability low; MSS: Microsatellite stable; MMR: Mismatch repair; NER: Nucleotide excision repair; NSCLC: Non-small cell lung cancer; OC: Ovarian cancer; PCNA: Proliferating cell nuclear antigen; RFC: Replicative factor C; SNP: Single nucleotide polymorphism; SCC: Squamous cell carcinoma; TCR: Transcription coupled repair; TLS: Translesion synthesis
\end{abstract}

\section{Introduction}

Cancer, characterized by uncontrolled growth and cell division, has commanded tremendous efforts from the research fraternity in elucidating all plausible pathways that drive a normal cell into the oncogenic pathway. Designing new strategies and protocols for treatment of the disease have been extensively studied yet unsuccessful due to the fact that the diagnosis happens at a very late stage of malignancy that leaves less scope for complete revival and high risk of relapse. Amongst the varied reasons of cancer development, mutations; either inherited in the germ line or arising from changes in DNA sequence of somatic tissues during the life, are the major instigators. These mutations may abnormally enhance the function of protooncogenes, or erase effects of the tumor suppressor gene (TSG) products. Over expressed protooncogenes over-ride cell senescence and silenced TSGs derail the control over cell division and genetic stability. TSGs controlling cell growth are critical; however, genes involving DNA repair systems hold an equally important value in maintaining the cellular integrity and growth.

The maintenance of the integrity of the information in DNA molecules is of utmost importance to the survival of a particular organism as well as to survival of the species [1]. Therefore, surviving species have evolved mechanisms for repairing DNA damage occurring as a result of either replication errors or environmental insults. Mutations occur more frequently in precancerous and cancerous cells than normal cells that result in genomic instability which is a driving force for tumorigenesis. Tumorigenesis acquires many genetic alterations on a chromosome and/or gene or at the nucleotide level. 


\section{Origin and fate of mutations}

Mutations in DNA have been implicated in degenerative processes such as aging, carcinogenesis, or genetic disorders [2]. A small fraction of these mutations may be due to replication errors. The major responsibility for the fidelity of replication resides in the specific pairing of nucleotide bases by the DNA polymerase and proofreading and excision of a misincorporated base. Additional accuracy is mediated by certain accessory proteins and post replication controls performed by DNA mismatch repair (MMR) mechanism. DNA damage that occur during replication may be simple incorporations of non-complementary bases leading to a point mutation, or a slippage event causing a frameshift mutation. Mutations may also arise spontaneously by deamination of bases or the loss of a base by active oxygen species that risk DNA integrity [1]. Several exogenous DNA-damaging agents may form premutagenic lesions by reacting with DNA bases, altering its chemical structure and halting the activity of DNA polymerase.

The tolerance of DNA lesions can be achieved through: damage avoidance (DA) and translesion synthesis (TLS). The DA process tends to ensure replication of a damaged template while avoiding the polymerase to replicate through the lesion. This can be achieved by post replication recombinational repair or by a polymerase strand switch and is intrinsically error free [3]. TLS requires that a polymerase reads through the damaged base at the expense of replicational accuracy and is thus error prone in essence.

\section{DNA repair mechanisms}

A collective action of different repair mechanisms is required for maintaining the integrity and genetic stability of a cell. The Base excision repair (BER) proteins excise and replace damaged DNA bases, mainly arising from endogenous oxidative and hydrolytic decay of DNA [4]. DNA glycosylases initiate this process by releasing the modified base followed by cleavage of the sugar phosphate chain, excision of the abasic residue and local DNA synthesis and ligation. A few unusual enzymes reverse rather than excise DNA damage. The human methyl guanosine methyl transferase (MGMT) removes methyl groups and other small alkyl groups from the $\mathrm{O}^{6}$ position of guanine. The nucleotide excision repair (NER) mainly removes bulky adducts caused by environmental agents. The human cells use an elaborate assembly of gene products to carry out NER like the XPC complex, DDB complex, XPA, and RPA proteins that bind to sites of DNA damage [4]. The unwinding of damaged DNA is brought about by two DNA helicases XPB and XPD and excision by XPG and ERCC1-XPF proteins. The mismatch repair (MMR) corrects occasional errors of DNA replication as well as heterologies formed during recombination. These genes encode proteins responsible for identifying mismatches and strand discrimination brought about by orientation of components of DNA replication complex such as PCNA. The DNA doublestrand breaks may be rectified by either homologous or non homologous recombination pathways. Homologous recombination involves branch migration enzymes and resolvases, while non homologous end-joining recombination involves protein factors like DNA-dependant protein kinase. These mechanisms exploit the redundancy of information inherent in the double helical DNA structure. The defective region in one strand can be returned to its original form by relying on the complementary information stored in the unaffected strand.

\section{The DNA mismatch repair mechanism}

The MMR system maintains genomic stability by repairing base-base mismatches and insertion/deletion loops (IDLs) originating during DNA replication, as well as heteroduplexes occurring during recombination. MMR improves the fidelity of DNA biosynthesis by 100-1000-fold, complementing the intrinsic error-free and proofreading properties of replicative DNA polymerases and lowering the overall mutation rate to one error per $10^{10}$ nucleotides synthesized [5].

At least six different MMR proteins are required to correct the mismatched DNA. For mismatch recognition, the MSH2 protein forms a heterodimer with either MSH6 or MSH3 (Mut S) depending on the type of lesion to be repaired (MSH6 is required for the correction of single-base mispairs, whereas both MSH3 and MSH6 may contribute to the correction of IDLs). A heterodimer of MLH1 and PMS2 (Mut L) coordinates the interplay between the mismatch recognition complex and other proteins necessary for MMR that include exonuclease 1 (EXO1), a helicase(s), proliferating cell nuclear antigen (PCNA), single-stranded DNA-binding protein (RPA) and DNA polymerases $\delta$ and $\varepsilon$. MLH1 may heterodimerize with two additional proteins, MLH3 and PMS1. PMS2 is required for the correction of single-base mismatches and PMS2 and MLH3 both contribute to the correction of IDLs, whereas the role of PMS1 in MMR awaits further research.

MMR also participates in transcription-coupled repair pathway, cell-cycle regulation and p53-dependent apoptotic response to a variety of DNA damage [6,7]. Alterations in the accurate mechanisms of MMR have profound consequences, from an increase of the mutation rate (mutator phenotype) to a pathogenic role in hereditary and sporadic cancer in humans [8].

\section{Structural insights of MMR proteins}

The Mut S dimer has a general shape of two 'opposing commas' or 'praying hands' joined by interactions at a single, composite ATP-binding site on one side and at the bound mispaired/unpaired DNA on the opposite side, immediately suggesting allosteric communication between DNA binding and ATP binding and hydrolysis. The dimer encircles two channels, one of which is occupied by the mismatched DNA $[9,10]$. 
Each subunit is formed by five flexible domains ensuring versatility in DNA damage recognition. Mismatch binding involves conformational changes in each monomer and a kinking of the mismatched DNA towards the major groove, widening and flattening the minor groove $[9,10]$. The five domains are defined as I - mismatch binding; II - connector; III - core; IV - DNA clamp and $\mathrm{V}$ - ATPase. The mismatch binding and DNA clamp domains are involved in binding to mismatched DNA, the connector and core domains constitute the backbone of each subunit and are involved in transmitting allosteric information of bound DNA co-factor to the ATPase domain $[9,10]$.

In both structures, the same FXE motif in domain I, is involved in binding to the G: T mismatch and to the unpaired T. Only one subunit interacts with the mismatched T, creating an asymmetry in the dimer. This asymmetry is reflected by the heterodimers MSH2-MSH6 and MSH2-MSH3 which suggests that MSH6 and MSH3 are involved in mismatched/misaligned DNA recognition. The ATPase domains are located on the opposite sides of the dimer, away from the bound DNA and are interleaved with each other. They contain the Walker A and B motifs, and their general fold resembles that of the ABC super family of ATPases. Each ATPase active site is shared between the two subunits across the dimer interface, to coordinate conformational changes of both monomers [9-11].

Mut L homologue has conserved C-terminal region that mediates constitutive dimerization and the N-terminal region undergoes ATP/ADP-regulated dimerization during MMR. Mut L is an elbow shaped two-domain monomer that resembles DNA gyrase and the chaperone HSP90. Upon binding to the non hydrolyzable ATP analogue, ADPnP, Mut L dimerizes and a large DNA binding groove is formed in the middle of the dimer. Dimerization is mediated by the association of the first domains, each binding a nucleotide molecule. Upon ADPnP- dimerization, two potential interfaces for interaction with Mut S and Mut $\mathrm{H}$ are formed. Mut $\mathrm{H}$ structure has homology to type II restriction endonucleases. It has a shape of a two domain open clamp delimiting a DNA binding cleft. The dimerization leads to ATP hydrolysis, generating an ADP-bound dimer. Mut L is also regulated by cycles of ATP binding and hydrolysis [12].

\section{Signalling complexes in DNA repair}

In the first model (sliding clamp), MSH2-MSH6 is capable of high-affinity mismatch recognition in its ADP-bound state and mismatch binding acts as nucleotide exchange factor provoking the ADP-ATP exchange. The ATP-bound undergoes a conformational change into a clamp with reduced affinity for the mismatch and diffuses freely along the DNA in an ATP hydrolysis-independent fashion (sliding clamp), signalling to additional components of the MMR $[13,14]$. Signalling to the replicative polymerase may change the polymerase's conformation and stops the forward processivity and activates the 3'-5' exonuclease activity to remove the newly synthesized DNA strand [13,14]. This hypothetical model explains the directionality of the excision towards the mismatch. Tethering of the MMR machinery to the replicative polymerase would allow strand discrimination. The direct interaction of MLH1, MSH3 and MSH6 with the PCNA may link MMR to DNA replication by marking the newly synthesized DNA [5].

A second model (ATP-dependent translocation) is based on binding of Mut S to heteroduplex DNA. Mut S extrudes a DNA loop in an ATP hydrolysis-dependent manner generating $\alpha$ - or $\Omega$-shaped structures with the mismatch located within the loop [5]. The recognition of mismatched DNA by Mut Sa does not require ADP binding. ATP binding then stimulates dissociation of Mut Sa from the mismatch and ATP hydrolysis provides the energy for translocation of Mut Sa along the helix contour [15].

A third model is based on the sharp kinking of mismatched DNA and conformational changes of Mut S upon DNA binding (induced fit). In this model Mut $S$ does not leave the mismatch and marks strand discrimination via direct protein-protein interactions with other MMR proteins such as Mut L and Mut $\mathrm{H}[9,10]$. In the presence of Mut L and ATP, Mut S can activate Mut $\mathrm{H}$-mediated cleavage in trans, i.e. the mismatch and the hemimethylated GATC site can reside on two separate DNA molecules [11]. In this model, the requirement of ATP is explained as a 'proofreading' means to verify mismatch recognition by Mut S, as ATP binding reduces the Mut $S$ affinity for homoduplex DNA more than for heteroduplex DNA. ATP would also authorize subsequent repair events, as only ATP binding by Mut S would allow productive interaction with Mut L [11].

Mut L dimer interacts with Mut S transmitter region: through this region, the conformational changes of concomitant ATP and mismatch DNA binding can be signalled to Mut L. In turn, Mut L can signal to Mut H via structural changes that depend on ATP hydrolysis [11].

\section{Additional interactions of MMR}

Proliferating cell nuclear antigen (PCNA) is an auxiliary factor for DNA polymerase $\delta$ and $\varepsilon$ and is necessary for DNA replication. It is a doughnut-shaped heterodimer that is loaded onto DNA by replicative factor C (RFC) in an ATP-dependent process and acts as a sliding clamp that encircles template DNA and provides processivity to the associated DNA polymerase. PCNA is emerging as a key player linking early and late events during MMR and possibly mediating strand discrimination. PCNA was found to interact with MSH3 and MSH6 and increases the specificity of mismatch recognition [16].

After MMR initiation, additional protein-protein interactions mediate subsequent repair steps. A direct interaction between 5'3' exonuclease EXOI/HEXI and MSH2 has been detected play a role in MMR. Polymerase $\delta$ and $\varepsilon$ effect DNA resynthesis. This process may be regulated by DNA interactions between MMR proteins and PCNA and RPA [17]. Finally, DNA ligase I, seals the nick produced by DNA polymerase $\delta$ and PCNA [5]. 


\section{MMR defects cause cancer}

Malfunction of MMR determine a status of genomic instability that is associated with predisposition to cancer. This may manifest itself as highly elevated rates of subtle mutations (microsatellite instability; MSI) throughout the genome [18]. Although MMRdeficient cells typically have a diploid or near-diploid DNA content, loss of heterology dependent suppression of recombination in these cells may promote gene conversion and expose tumor suppressor genes in analogy to loss of heterozygosity, or allow chromosomal translocations to occur. Increased mutational inactivation of genes involved in DNA double-strand break repair may contribute to an elevated degree of chromosomal aberrations in MMR-deficient cells. Critical genes may be affected with mutations, conferring a growth advantage on the cells $[19,20]$.

Germline mutations of MMR genes are found in families affected with hereditary non polyposis colorectal cancer (HNPCC) or Lynch syndrome, an autosomal dominant disease predisposing to cancer of the colon, endometrium, stomach, ovary, urinary and biliary tracts [21]. Somatic mutations of MMR genes and epigenetic inactivation of MLH1 are also found in 12-15\% of sporadic colorectal cancer cases and in a variable fraction of cancers of the endometrium, stomach and other sites. Due to unrepaired slippage intermediates originated during replication of simple repetitive sequences, tumors from HNPCC individuals and sporadic MMR-defective tumors manifest MSI [22].

Pathogenic mutations of MMR genes often involve frameshift-inducing insertions/deletions, non-sense mutations, or changes in critical residues directly affecting catalytic (ATP binding/hydrolysis) or DNA binding activity. Disease-causing missense mutations are pathogenic because they disrupt critical protein-protein interactions during MMR. Several MLH1 missense mutations found in HNPCC kindred's cluster in the C-terminal region of the protein and impair the interaction with PMS2 [23].

\section{Role of MMR in epigenetic modification}

Epigenetic inactivation mainly the DNA promoter methylation of DNA repair genes in cancer has been reported in several DNA repair pathways. It has been shown that methylation in the promoter region of $M L H 1$ correlates with decreased activity of the gene. This gene is epigenetically inactivated in other types of cancers other than HNPCC, in sporadic endometrial carcinoma, gastric cancers, sporadic colorectal carcinoma (CRC), ovarian tumors (OC), NSCLC, oral squamous cell carcinoma (SCC), neck SCC, and acute myeloid leukemia (AML) [24-33]. Other MMR genes are also controlled by promoter methylation. $\mathrm{MSH} 2$ is methylated in CRC, primary non-small cell lung carcinoma (NSCLC), oral SCC, OC and neurofibromatosis type 1 [34-37]. MSH6 methylation occurs in CRC and MSH3 methylation was found in sporadic CRC [38]. In conclusion, methylation of the gene MLH1 may have considerable importance in cancer development and as a prognostic factor and the genes MSH2, MSH3 and MSH6 are interesting candidates as well.

\section{Role of MMR in microsatellite instability}

Microsatellites refer to long segments of non-transcribed DNA that are composed of a repeating mononucleotide or dinucleotide sequences. These long DNA sequences provide a simple indicator of genetic mutation rate and risk because mutations are readily apparent in these long repeating sequences. Microsatellite instability is commonly tested at five loci (BAT 25, BAT 26, D2S123, D5S346 and D17S250), the best known of which are BAT 25 and BAT 26 which consists of 25 and 26 adenine nucleotides in a row respectively. The test sites for MSI are not involved in the carcinogenic process. Thirty two genes in the human genome have mononucleotide repeats of more than 7 elements [39]. These long repetitive sequences are believed to be more prone to mutation than non-repetitive sequences. A number of these genes have been shown to be altered in MSI+ colorectal tumors. They encode proteins involved in signal transduction (TGF $\beta$-RII, IGFIIR, PTEN), apoptosis and inflammation (BAX, caspase-5), transcription regulation (E2F4, TCF-4), and DNA repair (MSH6, MSH3, MLH3, MED-1, RAD50, DNA-PKcs, BLM).

Tumours are classified as having high frequency microsatellite instability (MSI-H) if $>30 \%$ of the markers show instability. If $<30 \%$ of markers show instability, the tumour is classified as low-frequency microsatellite instability (MSI-L), while if there is no apparent instability, the tumour is classified as microsatellite stable (MSS) [40].

MSI is found in more than $90 \%$ of the HNPCC tumors, it is also seen in 15-20\% of the sporadic CRCs [41]. MSI also affects other epithelial tumors including the stomach, endometrium, and ovary. MSI among endometrial cancers varies from $17 \%$ to $32 \%$ in sporadic cancers while the frequency increases to more than $75 \%$ of those tumors associated with HNPCC [42]. For ovarian cancers, MSI range is detected in up to 50\% cases [43]. MSI may serve as a marker for the identification of a mutator phenotype.

In spite of extensive studies, the clinical significance of the MSI+ phenotype remains a matter of debate. MSI+ colorectal tumors are associated with a better survival and a reduced likelihood of metastasis. Several studies have reported that the MSI+ phenotype may influence the outcome of chemotherapy $[44,45]$.

\section{Role of MMR in genetic polymorphisms}

Single nucleotide polymorphisms (SNPs) are DNA variants where a single nucleotide at a fixed position in the genome is substituted with another. SNPs can be used to measure admixture in populations and may be utilized to map genes that could account for the differences in disease incidence between populations [46]. Exonic SNPs result in amino acid substitutions that may affect mRNA stability and alter splicing signals in genes [47]. SNPs in introns, regulatory and gene-distant regions affect gene regulation by splicing mechanism [48]. Promoter SNPs can directly affect transcription of a gene. 
Polymorphisms in MMR genes may primarily be associated with CRC. A number of MMR SNPS have been evaluated in CRC and many reported are with extra colonic where MSI status was not evaluated. The MLH1 I219V polymorphism, located in exon 8 at nucleotide position $655(\mathrm{~A}>\mathrm{G})$, was shown to be associated with an increased risk for childhood acute lymphoblastic leukemia [49]. Few studies have found a significant association between the I219V homozygous variant (GG) and an increased risk of breast cancer [50]. The MSH2 G322D polymorphism is located in the coding-region of the MSH2 gene, in exon 6, at nucleotide position 965 (G>A). This alteration results in a modest decrease in mismatch repair efficiency [51]. Another MSH2 SNP, IVS12-6T>C has been associated with acute myeloid leukemia, non-Hodgkin lymphoma, familial and sporadic CRCs, high-grade dysplasia and cancer in ulcerative colitis patients, and primary lung cancer [52]. The MSH6 G39E, located in exon 1, (116G>A) was shown to be associated with increased risk of both colon and rectal cancers [53]. One of the most studied MMR polymorphisms is MLH1 $\mathrm{D} 132 \mathrm{H}$, which was associated with susceptibility to sporadic CRCs [54]. This polymorphism attenuates the ATPase activity of MLH1 without affecting the MMR functions. This uncoupling of the MLH1 functions result in increased risk to MSS, but not MSI-H CRCs [54].

\section{Germline mutations of MMR genes}

The $\mathrm{MSH} 2$ gene consists of 15 exons, located on chromosome 2p22-p21, and encodes a 934-amino acid protein The mutations of $\mathrm{MSH} 2$ represent approximately $40 \%$ of the mutations detected in HNPCC. Among the 154 different $M$ SH2 mutations that have been analyzed, about $80 \%$ lead to premature termination of the $M S H 2$ gene product. Single-base substitutions directly resulting in a stop codon account for one-fourth of the truncating mutations, whereas half are due to small insertions or deletions, causing frameshift generating downstream termination codons. Large genomic deletions, which account for the remaining truncating mutations, represent a frequent cause of HNPCC, with some of them showing inter-ethnic differences [55].

The MLH1 locus is located on chromosome 3p21.3, consists of 19 exons, and encodes a 756-amino acid protein. 200 different mutations of $M L H 1$ have been described in the patients with HNPCC, representing 55\% of the mutations reported so far. A cluster of $\mathrm{MLH} 1$ mutations has been found in the region spanning exons 15 and 16. Most of the MLH1 mutations identified result in a truncated gene product by either frameshift or splice site mutations leading to exon deletion [56].

The MSH6 locus spans $27 \mathrm{~kb}$ on chromosome 2p16, consists of 11 exons and encodes a 1360-amino acid protein. The frequency of MSH6 germline mutations has been evaluated in various population-based series of colorectal cancer, including sporadic cases, familial non-HNPCC cases, as well as classical HNPCC cases, early-onset colorectal cancers and tumors with low levels of MSI

The MSH3 gene locus spans $243 \mathrm{~kb}$ on chromosome 5q11-q12, consists of 27 exons that encode a 1128-amino acid protein. The role of MSH3 in the initiation of colorectal carcinogenesis should be regarded as marginal. The PMS1 locus consists of 13 exons spanning about $161 \mathrm{~kb}$ on chromosome $2 \mathrm{q} 31-\mathrm{q} 33$, and encodes a 932-amino acid protein. The PMS1 mutation is a rare event in colorectal carcninogenesis. The $M L H 3$, a 1429-amino acid protein, mapped to chromosome 14q24.3, a region showing frequent $\mathrm{LOH}$ in sporadic CRC [58]. Future studies are required to assess the role of $M L H 3$ in the progression of colorectal carcinogenesis.

The PMS2 genomic locus encompasses $33 \mathrm{~kb}$ on chromosome 7p22, consists of 14 exons, and encodes an 862-amino acid protein. A non-expressed pseudogene has been described in the region of PMS2 and polymorphisms in this region may be mistaken for mutations [59]. The role of PMS2 in familial colorectal cancer is highly questionable, except in the Turcot's variant of the disease.

\section{Role of MMR in platinum resistance}

Platinum compounds such as cisplatin, carboplatin and oxaliplatin are non-classic alkylating agents frequently used in the treatment of cancer of the ovary, testis, bladder, head and neck, cervix, lung, bone and nervous system [60]. Unfortunately, resistance has limited the efficacy of these agents in most diseases. Resistance to platinum-based chemotherapy can be intrinsic or acquired. Increased DNA repair activity is an important factor in acquired platinum resistance in cancer cells. In the case of platinum resistance, the DNA repair system is diverted to the repair of platinum-induced damage intended to kill tumor cells. Platinum adducts are repaired by a process of 'cutting, removing, and replacing' that involves many proteins in the NER pathway [61,62].

A functional MMR system is required for the detection of damaged DNA created by cisplatin and carboplatin. MMR proteins can bind to DNA-adducts created by platinum drugs and repair the DNA conferring damage thus conferring sensitivity to treatment [63]. Platinum complexes interfere with normal MMR activity and lead the cell to apoptosis. When MMR is deficient, cells can continue to proliferate in spite of DNA damage caused by platinum drugs and are thus resistant. MMR-deficient cells that have lost MMR function or that lack expression of MLH1 or MSH2 become resistant to DNA-damaging platinum compounds due to futile repair [64].

\section{MMR and other DNA transactions}

In addition to a direct role in MMR, mammalian MMR proteins are involved in several DNA transactions, including transcriptioncoupled repair (TCR), base excision repair (BER) and recombination and meiosis. NER is involved in the removal of UV lightinduced pyrimidine dimers to adduct generated by benzo[a]pyrene and cisplatin [1]. TCR is a NER pathway that preferentially 
corrects lesions in the transcription template strand of RNA polymerase II-transcribed genes [65.] Although the mechanistic details of the involvement of MMR proteins in TCR are not known, specific interactions between MSH2 and NER proteins have been detected in yeast [66]. MSH2 contributes to the recognition of a stalled RNA polymerase on the transcription template strand at sites of DNA damage. Also, MSH2-MSH6 heterodimer can bind to some lesions repaired by NER. MMR proteins may function during resynthesis of the transcribed strand [67]. This suggests that inactivation of MMR function may lead to accumulation of both environmental and endogenous oxidative DNA lesions.

The tumor suppressor genes BRCA1 and BRCA2 are responsible for the majority of the cases of hereditary predisposition to breast and ovarian cancer. They play a role in the repair of DNA double-strand breaks. BRCA1 interacts directly with $M S H 2$, MSH3 and MSH6 and indirectly with MLH1 and PMS2 in addition to ATM, BLM and RAD50-MRE11-NBS1 to form BASC (BRCA1 associated genome surveillance complex) [68]. The heterodimer MSH2-MSH6, provide a DNA damage recognition signal required for the BRCA1 and BRCA2 dependent TCR [68].

MLH1 provides a link between MMR and BER. BER repairs endogenous oxidative lesions caused by reactive oxygen species and exogenous lesions such as alkylation of purines and formation of adducts [69]. BER acts on mismatches via the action of mismatch specific DNA N-glycosylases [70]. The human MED1 protein contains a N-terminal 5-methyl cytosine binding domain (MBD) and a C-terminal catalytic region. This interaction implies unprecedented role of DNA methylation.

The molecular mechanisms underlying suppression of genetic recombination by MMR in mammalian cells are less clear and the relevant interactions with recombination specific factors are not established. The human Bloom syndrome helicase (BLM), a component of BASC, was shown to directly interact with MLH1 [71]. It has been hypothesized that MSH4-MSH5 and MLH1MLH3 complexes promote the formation and stabilization of Holliday junctions and their preferential resolution into cross-overs rather than non cross-overs [72].

\section{Clinical implications}

DNA mismatch repair defects are common in several cancers as inferred from the occurrence of MSI. MSI analysis and/or immunohistochemical staining for MMR protein expression offer useful preliminary tests for MMR gene involvement [73]. The detection of a MMR defect may provide an important piece of information to guide the clinical management of the patients. Malfunction of the MMR system may modify the response to the cytotoxic drug fluorouracil, a competitive inhibitor for substrates critical for DNA synthesis. This works in concert with MMR deficiency and enhances apoptosis in these cells [74]. The resistance of MMR-deficient cells to alkylating agents is thought to result from the failing detection of alkylation adducts and impaired induction of apoptosis [75]. Increased knowledge of the MMR system and its connection to other biologic pathways is essential for better understanding of the fundamental mechanisms of cancer development and to identify targets for preventive and therapeutic interventions.

\section{Conclusions and future perspective}

MMR has entered the centre stage of cancer research and molecular medicine with discoveries of its role in the pathogenesis of HNPCC and resistance to alklylating agents and other anti-neoplastic drugs. Inactivation of MMR either due to germline mutations or hypermethylation of $M L H 1$ promoter is closely associated with cancer predisposoition. MMR proteins are engaged in a complex network of molecular interactions that extends to proteins that participate in recombination, transcription-coupled repair and base excision repair. MMR proteins repair ephemeral DNA lesion, mismatch formed by chemically normal bases. Excessive damage activates apoptosis in a MMR-dependent fashion. Thus, the complexity of the interactions of MMR components is a reflection of their central role in mutation avoidance and cell death regulation.

In future, efforts should be directed towards novel molecular approaches to cancer prevention and treatment that build on our increased understanding of MMR function. Chemopreventive agents should be developed that specifically target precancerous cells in which a defect in MMR is propelling them to malignancy. Novel radio and chemotherapeutic strategies will have to take into account the MMR deficiency of the individual tumor. MMR deficient malignancies should be treated preferentially with frameshift-inducing agents and other drugs for which increased sensitivity is brought about by a MMR defect.

\section{References}

1. Lindahl T, Wood RD (1999) Quality control by DNA repair. Science 286: 1897-905.

2. Jackson AL, Loeb LA (1998) The mutation rate and cancer. Genetics 148: 1483-90.

3. Higgins NP, Kato K, Strauss B (1976) A model for replication repair in mammalian cells. J Mol Biol 101: 417-25.

4. Fishel R, Kolodner RD (1995) Identification of mismatch repair genes and their role in the development of cancer. Curr Opin Genet Dev 5: $382-95$.

5. Jiricny J (1998) Replication errors: challenging the genome. EMBO J 17: 6427-36.

6. Wu J, Gu L, Wang H, Geacintov NE, Li GM (1999) Mismatch repair processing of carcinogen-DNA adducts triggers apoptosis. Mol Cell Biol 19: 8292-301.

7. Zhang H, Richards B, Wilson T, Lloyd M, Cranston A, et al. (1999) Apoptosis induced by over expression of hMSH2 or hMLH1. Cancer Res 59: 3021-7. 
8. Lynch HT, de la Chapelle A (1999) Genetic susceptibility to non-polyposis colorectal cancer. J Med Genet 36: 801-18.

9. Obmolova G, Ban C, Hsieh P, Yang W (2000) Crystal structures of mismatch repair protein Mut S and its complex with a substrate DNA. Nature 407: 703-10.

10. Lamers MH, Perrakis A, Enzlin JH, Winterwerp HH, de Wind N, et al. (2000) The crystal structure of DNA mismatch repair protein Mut S binding to a G: T mismatch. Nature 407: 711-7.

11. Junop MS, Obmolova G, Rausch K, Hsieh P, Yang W (2001) Composite active site of an ABC ATPase: MutS uses ATP to verify mismatch recognition and authorize DNA repair. Mol Cell 7: 1-12.

12. Ban C, Junop M, Yang W (1999) Transformation of MutL by ATP binding and hydrolysis: a switch in DNA mismatch repair. Cell 97: 85-97.

13. Gradia S, Subramanian D, Wilson T, Acharya S, Makhov A, et al. (1999) hMSH2-hMSH6 forms a hydrolysis-independent sliding clamp on mismatched DNA. Mol Cell 3: 255-61.

14. Fishel R (1999) Signalling mismatch repair in cancer. Nat Med 5: 1239-41.

15. Blackwell LJ, Martik D, Bjornson KP, Bjornson ES, Modrich P (1998) Nucleotide-promoted release of hMutSa from heteroduplex DNA is consistent with an ATP-dependent translocation mechanism. J Biol Chem 273: 32055-62.

16. Kleczkowska HE, Marra G, Lettieri T, Jiricny J (2001) hMSH3 and hMSH6 interact with PCNA and colocalize with it to replication foci. Genes Dev 15: 724-36. 17. Schmutte C, Marinescu RC, Sadoff MM, Guerrette S, Overhauser J, et al. (1998) Human exonuclease I interacts with the mismatch repair protein hMSH2. Cancer Res 58: 4537-42.

18. Eshleman JR, Lang EZ, Bowerfind GK, Parsons R, Vogelstein B, et.al. (1995) Increased mutation rate at the hprt locus accompanies microsatellite instability in colon cancer. Oncogene 10: 33-7.

19. Kim NG, Choi YR, Baek MJ, Kim YH, Kang H, et.al. (2001) Frameshift mutations at coding mononucleotide repeats of the hRAD50 gene in gastrointestinal carcinomas with microsatellite instability. Cancer Res 61:36-8.

20. Giannini G, Ristori E, Cerignoli F, Christian R, Massimo Z, et.al. (2002) Human MRE11 is inactivated in mismatch repair-deficient cancers. EMBO Rep 3: 248-54.

21. Boland CR (2000) Molecular genetics of hereditary nonpolyposis colorectal cancer. Ann NY Acad Sci 910: 50-61.

22. Dietmaier W, Wallinger S, Bocker T, Kullmann F, Fishel R, et al. (1997) Diagnostic microsatellite instability: definition and correlation with mismatch repair protein expression. Cancer Res 57: 4749-56.

23. Guerrette S, Acharya S, Fishel R (1999) The interaction of the human MutL homologues in hereditary nonpolyposis colon cancer. J Biol Chem $274: 6336-41$.

24. Kane MF, Loda M, Gaida GM, Lipman J, Mishra R, et al. (1997) Methylation of the hMLH1 promoter correlates with lack of expression of hMLH1 in sporadic colon tumors and mismatch repair-defective human tumor cell lines. Cancer Res 57: 808-11.

25. Esteller M, Levine R, Baylin SB, Ellenson LH, Herman JG (1998) MLH1 promoter hypermethylation is associated with the microsatellite instability phenotype in sporadic endometrial carcinomas. Oncogene 17: 2413-17.

26. Fleisher AS, Esteller M, Wang S, Tamura G, Suzuki H, et al. (1999) Hypermethylation of the hMLH1 gene promoter in human gastric cancers with microsatellite instability. Cancer Res 59: 1090-5.

27. Herman JG, Umar A, Polyak K, Graff JR, Ahuja N, et al. (1998) Incidence and functional consequences of hMLH1 promoter hypermethylation in colorectal carcinoma. Proc Natl Acad Sci USA 95: 6870-75.

28. Gras E, Catasus L, Arguelles R, Moreno-Bueno G, Palacios J, et al. (2001) Microsatellite instability, MLH1 promoter hypermethylation, and frameshift mutations at coding mononucleotide repeat microsatellites in ovarian tumors. Cancer 92: 2829-36.

29. Wang YC, Lu YP, Tseng RC, Lin RK, Chang JW, et al. (2003) Inactivation of hMLH1 and hMSH2 by promoter methylation in primary non-small cell lung tumors and matched sputum samples. J Clin Invest 111: 887-95.

30. Czerninski R, Krichevsky S, Ashhab Y, Gazit D, Patel V, et al. (2009) Promoter hypermethylation of mismatch repair genes, hMLH1 and hMSH2 in oral squamous cell carcinoma. Oral Dis 15: 206-13.

31. Liu K, Huang H, Mukunyadzi P, Suen JY, Hanna E, et al. (2002) Promoter hypermethylation: an important epigenetic mechanism for hMLH1 gene inactivation in head and neck squamous cell carcinoma. Otolaryngol Head Neck Surg 126: 548-53.

32. Steinmann K, Sandner A, Schagdarsurengin U, Dammann RH (2009) Frequent promoter hypermethylation of tumor-related genes in head and neck squamous cell carcinoma. Oncol Rep 22: 1519-26.

33. Seedhouse CH, Das-Gupta EP, Russell NH (2003) Methylation of the hMLH1 promoter and its association with microsatellite instability in acute myeloid leukemia. Leukemia 17: 83-8.

34. Lawes DA, Pearson T, Sengupta S, Boulos PB (2005) The role of MLH1, MSH2 and MSH6 in the development of multiple colorectal cancers. BJC 93: 472-7.

35. Nagasaka T, Rhees J, Kloor M, Gebert J, Naomoto Y, et al (2010) Somatic hypermethylation of MSH2 is a frequent event in Lynch syndrome colorectal cancers. Cancer Res 70: 3098-108.

36. Zhang H, Zhang S, Cui J, Zhang A, Shen L et al (2008) Expression and promoter methylation status of mismatch repair gene hMLH1 and hMSH2 in epithelial ovarian cancer. Aust NZ J Obstet Gynaecol 48: 505-9.

37. Titze S, Peters H, Wahrisch S, Harder T, Guse K, et al. (2010) Differential MSH2 promoter methylation in blood cells of Neurofibromatosis type 1 (NF1) patients. Eur J Hum Genet 18: 81-7.

38. Benachenhou N, Guiral S, Gorska-Flipot I, Michalski R, Labuda D, et al. (1998) Allelic losses and DNA methylation at DNA mismatch repair loci in sporadic colorectal cancer. Carcinogenesis 19: 1925-29.

39. Duval A, Hamelin R (2002) Mutations at coding repeat sequences in mismatch repair-deficient human cancers: toward a new concept of target genes for instability. Cancer Res 62: 2447-54.

40. Boland CR, Thibodeau SN, Hamilton SR, Sidransky D, Eshleman JR, et al. (1998) A National Cancer Institute Workshop on Microsatellite Instability for cancer detection and familial predisposition: development of international criteria for the determination of microsatellite instability in colorectal cancer. Cancer Res 58: 5248-57.

41. Liu B, Nicolaides NC, Markowitz S, Willson JK, Parsons RE, et al. (1995) Mismatch repair gene defects in sporadic colorectal cancers with microsatellite instability. Nat Genet 9: 48-55. 
42. Ichikawa Y, Lemon SJ, Wang S, Franklin B, Watson P, et al. (1999) Microsatellites instability and expression of hMSH2 or hMLH1 in normal and malignant endometrial and ovarian epithelium in hereditary nonpolyposis colorectal cancer family members. Cancer Genet Cytogenet 112: 2-8.

43. Arzimanglou IL, Lallas T, Osborne M, Barber H, Gilbert F (1996) Microsatellite instability differences between familial and sporadic ovarian cancer. Carcinogenesis 17: 1799-1804.

44. Hemminki A, Mecklin JP, Jarvinen H, Aaltonen LA, Joensuu H (2000) Microsatellite instability is a favorable prognostic indicator in patients with colorectal cancer receiving chemotherapy. Gastroenterology 119: 921-8.

45. Elsaleh H (2001) The microsatellite instability phenotype in human colorectal carcinoma: relationship to sex, age, and tumor site. Gastroenterology 121: 230-1. 46. Shriver MD, Mei R, Parra EJ, Sonpar V, Halder I, et al. (2005) Large-scale SNP analysis reveals clustered and continuous patterns of human genetic variation. Hum Genomics 2: 81-9.

47. Chamary JV, Parmley JL, Hurst LD (2006) Hearing silence: non-neutral evolution at synonymous sites in mammals. Nat Rev Genet 7: 98-108.

48. Zanke BW, Greenwood CM, Rangrej J, Kustra R, Tenesa A, et al. (2007) Genome-wide association scan identifies a colorectal cancer susceptibility locus on chromosome 8q24. Nat Genet 39: 989-94.

49. Mathonnet G, Krajinovic M, Labuda D, Sinnett D (2003) Role of DNA mismatch repair genetic polymorphisms in the risk of childhood acute lymphoblastic leukaemia. Br J Haematol 123: 45-8.

50. Listgarten J, Damaraju S, Poulin B, Cook L, Dufour J, et al. (2004) Predictive models for breast cancer susceptibility from multiple single nucleotide polymorphisms. Clin Cancer Res 10: 2725-37.

51. Ellison AR, Lofing J, Bitter GA (2001) Functional analysis of human MLH1 and MSH2 missense variants and hybrid human-yeast MLH1 proteins in Saccharomyces cerevisiae. Hum Mol Genet 10: 1889-900.

52. Jung CY, Choi JE, Park JM, Chae MH, Kang HG, et al. (2006) Polymorphisms in the hMSH2 gene and the risk of primary lung cancer. Cancer Epidemiol Biomarkers Prev 15: 762-8.

53. Campbell PT, Curtin K, Ulrich C, Samowitz W, Bigler J, et al. (2009) Mismatch repair polymorphisms and risk of colon cancer, tumor microsatellite instability, and interactions with lifestyle factors. Gut 58: 661-7.

54. Lipkin SM, Rozek LS, Rennert G, Yang W, Chen PC, et al. (2004) The MLH1 D132H variant is associated with susceptibility to sporadic colorectal cancer. Nat Genet 36: 694-9.

55. Wijnen J, van der Klift H, Vasen H, Khan PM, Menko F, et al. (1998) MSH2 genomic deletions are a frequent cause of HNPCC. Nat Genet 20: 326-8.

56. Wijnen J, Khan PM, Vasen H, Menko F, van der Klift H, et al. (1996) Majority of hMLH1 mutations responsible for hereditary nonpolyposis colorectal cancer cluster at the exonic region 15-16. Am J Hum Gene.58: 300-7.

57. Huang J, Kuismanen SA, Liu T, Chadwick RB, Johnson CK, et al. (2001) MSH6 and MSH3 are rarely involved in genetic predisposition to nonpolypotic colon cancer. Cancer Res 61: 1619-23.

58. Lipkin SM, Wang V, Jacoby R, Banerjee-Basu S, Baxevanis AD, et al. (2000) MLH3: a DNA mismatch repair gene associated with mammalian microsatellite instability. Nat Genet 24: 27-35.

59. Chadwick RB, Meek JE, Prior TW, Peltomaki P, de La Chapelle A (2000) Polymorphisms in a pseudogene highly homologous to PMS2. Hum Mutat 16: 530.

60. O’Dwyer PJ, Stevenson JP, Johnson SW (2000) Clinical pharmacokinetics and administration of established platinum drugs. Drugs 59: 19-27.

61. Mueller MD (2008) Report on platinum drug symposium. West Virgin Med J 104: 8-9.

62. Bonetti A, Leone R, Muggia F, Howell SB (2009) Platinum and other heavy metal compounds in cancer chemotherapy: Molecular mechanisms and clinical applications. Humana press, New York, United States.

63. Wu J, Zhu BB, Yu J, Zhu H, Qiu L, et al. (2003) In vitro and in vivo modulations of benzo[c]phenanthrene-DNA adducts by DNA mismatch repair system. Nucleic Acids Res 31: 6428-34.

64. Bevilacqua RA, Simpson AJ (2000) Methylation of the promoter but no hMLH1 mutations in sporadic gastric carcinomas with high-level microsatellite instability. Int J Cancer 87: 200-3.

65. Leadon SA (1999) Transcription-coupled repair of DNA damage: unanticipated players, unexpected complexities. Am J Hum Genet 64: 1259-63.

66. Bertrand P, Tishkoff DX, Filosi N, Dasgupta R, Kolodner RD (1998) Physical interaction between components of DNA mismatch repair and nucleotide excision repair. Proc Natl Acad Sci USA 95: 14278-83.

67. Leadon SA, Avrutskaya AV (1997) Differential involvement of the human mismatch repair proteins, hMLH1 and hMSH2, in transcription-coupled repair. Cancer Res 57: 3784-91.

68. Wang Y, Cortez D, Yazdi P, Neff N, Elledge SJ, et al (2000) BASC, a super complex of BRCA1-associated proteins involved in the recognition and repair of aberrant DNA structures. Genes Dev 14: 927-39.

69. Krokan HE, Nilsen H, Skorpen F, Otterlei M, Slupphaug G (2000) Base excision repair of DNA in mammalian cells. FEBS Lett 476: 73-7.

70. Bellacosa A, Cicchillitti L, Schepis F, Riccio A, Yeung AT, et al. (1999) MED1, a novel human methyl-CpG binding endonuclease, interacts with the DNA mismatch repair protein MLH1. Proc Natl Acad Sci USA 96: 3969-74.

71. Langland G, Kordich J, Creaney J, Goss KH, Lillard-Wetherell K, et al. (2001) The Bloom's syndrome protein (BLM) interacts with MLH1 but is not required for DNA mismatch repair. J Biol Chem 276: 30031-5.

72. Nakagawa T, Datta A, Kolodner RD (1999) Multiple functions of MutS- and MutL-related heterocomplexes. Proc Natl Acad Sci USA 96: $14186-8$.

73. Lindor NM, Burgart LJ, Leontovich O, Goldberg RM, Cunningham JM, et al. (2002) Immunohistochemistry versus microsatellite instability testing in phenotyping colorectal tumors. J Clin Oncol 20: 1043-8.

74. Elsaleh H, Joseph D, Grieu F, Zeps N, Spry N, et al. (2000) Association of tumour site and sex with survival benefit from adjuvant chemotherapy in colorectal cancer. Lancet 355: 1745-50.

75. Fink D, Aebi S, Howell SB (1998) The role of DNA mismatch repair in drug resistance. Clin Cancer Res 4: 1-6. 


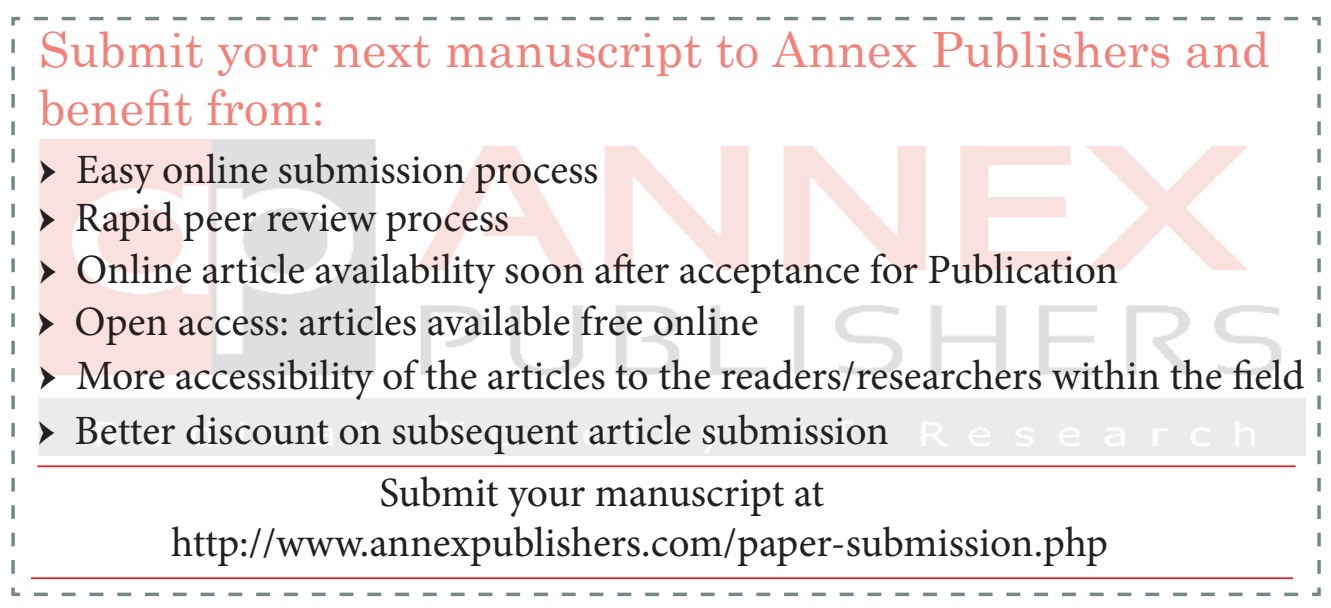

